\title{
Characterization of the interaction of staphylococcal enterotoxin B with CD1d expressed in human renal proximal tubule epithelial cells
}

Rasha Hammamieh ${ }^{1 *}$, Nabarun Chakraborty ${ }^{1}$, Yixin Lin², Jeffrey W Shupp ${ }^{3}$, Stacy-Ann Miller ${ }^{1}$, Sam Morris ${ }^{2}$ and Marti Jett ${ }^{1}$

\begin{abstract}
Background: Participation of renal cells in the pathogenesis of staphylococcal enterotoxin B (SEB) is critical for late cleansing and sequestration of the antigens facilitated by CD1d mediated antigen sensing and recognition. This is a noted deviation from the typical antigen recognition process that recruits the major histocompatibility complex class II (MHC II) molecules. The immunological importance of CD1d is underscored by its influences on the performances of natural killer T-cells and thereby mediates the innate and adaptive immune systems.

Results: Using diffraction-based dotReady ${ }^{\mathrm{TM}}$ immunoassays, the present study showed that SEB directly and specifically conjugated to CD1d. The specificity of the conjugation between SEB and CD1d expressed on human renal proximal tubule epithelial cells (RPTEC) was further established by selective inhibition of CD1d prior to its exposure to SEB. We found that SEB induced the expression of CD1d on the cell surface prompting a rapid conjugation between them. The mRNA transcripts encoding CD1d remained elevated potentially after completing the antigen cleansing process.

Conclusion: Molecular targets associated with the delayed pathogenic response have essential therapeutic values. Particularly in the event of bioterrorism, the caregivers are typically able to intervene much later than the toxic exposures. Given circumstances mandate a paradigm shift from the conventional therapeutic strategy that counts on targeting the host markers responding to the early assault of pathogens. We demonstrated the role of CD1d in the late stage of pathogen recognition and cleansing, and thereby underscored its clinical potential in treating bioweaponizable antigens, such as Staphylococcal enterotoxin B (SEB).
\end{abstract}

\section{Background}

$\mathrm{SEB}$, a member of the exotoxin family produced by staphylococci [1,2], is a superantigen (sAg) capable of inducing toxic shock through intranasal or intravenous portals. Transmittable via air, food and water, the SEBinduced toxemia causes pyogenic damage that manifests as immunological irregularities, arthritis, and autoimmune disease cascading to multi-organ dysfunction and lethal consequences $[1,3]$. Continued clinical interest

\footnotetext{
* Correspondence: Rasha.Hammamieh1.civ@mail.mil

${ }^{1}$ Integrative Systems Biology, US Army Center for Environmental Health Research Fort Detrick, 568 Doughten Drive, Fort Detrick, MD 21702-5010, USA

Full list of author information is available at the end of the article
}

about staphylococcal enterotoxins (SEs) is attributed to a general inadequacy of its effective treatment [3,4]. Many incidences alleging SEB as the chief food poisoning contaminant [5] and its easy adaptability to bioweapons [6] further justify its continued clinical relevance.

Administrations of the anti-toxin and anti-inflammatory agents are the typical clinical strategies available nowadays $[3,7]$. Conventional strategies targeting early pathogenic markers have faced some serious deficiencies particularly when they intervene long after the pathogenic assault [8]. This concern has been multiplied by the failure of the conventional therapeutic strategy in treating septic shock [9], which shares many patho-clinical similarities with toxic shock $[10,11]$. In the advent of chemical warfare, 
therapeutic interventions typically lag behind the toxic assault. Hence, the molecular signatures involved with the delayed response to bioweaponizable toxins, such as SEB, are of significant clinical interest.

Accumulating evidences suggest robust clinical efficacy in targeting the post-assault downstream candidates responsible for SEB pathogenesis [3,12]. In recent past, the focus of investigation for clinical targets has shifted from blood cells to the non-lymphoid cell types derived from kidney [13], spleen [14], lungs [15], and gut [16]. Recent findings demonstrating the translocation of bacterial infection from skin [17] to organs, such as kidney and spleen without hematic invasion, further enhanced the interests on such peripheral organs in this context. It was also noted that many cell types $[18,19]$ express atypical binding sites for SEB, which underscore the essentiality of cell/tissue-specific studies. In other words, CD1d (cluster of differentiation 1d glycoprotein) expressed on kidney cell surface may demonstrate a very unique response characteristics to SEB.

The critical role of the kidneys in exotoxin clearance and sequestration [20-25] was attested by identifying the renal excretion process as the primary route of removing staphylococcal enterotoxin A (SEA) from plasma [26]. Supporting evidence includes the non-human primate study reporting $\sim 70 \%$ accumulation of SEB in the renal proximal tubule epithelial cells (RPTECs) within $90 \mathrm{mi}-$ nutes of aerosol administration [22]. SEB introduced through a cutaneous burn wound of rat was found traversed to and localized in the kidney 6 days post-burn [27], and a murine genomic study showed a delayed response of renal genes after lethal SEB shock [12]. Further investigation of the renal response in SEB pathogenesis may help to identify an alternative avenue for clinical intervention.

The present study is focused on CD1d for two primary reasons. Firstly, CD1d selectively expressed in the renal epithelial tissues are recruited for antigen sensing, recognition and cleansing at the late stage of pathogenesis [13,28-31]. Unlike a typical host-mediated antigen recognition sequence, the intact sAgs are submitted to the antigen presenting cells (APC) by CD1d, a phylogenetic analog of major histocompatibility complex (MHC) class I and II molecules operating as an antigen-trafficking agent [32-34]. Secondly, CD1d controls the function of natural killer T-cells (NKT cells); the CD1d-NKT cellmediated sAg recognition event rapidly triggers the release of many cytokines, and thereby systematically influences the hosts' adaptive and innate immune systems $[35,36]$.

The involvement of CD1d in pathogenesis has been investigated in the past, primarily focused on the lymphoid cells [37-41], while the knowledge gap exists in comprehending the CD1d-mediated pathogenesis in kidney cells and its role in the late stage of pathogenesis. To bridge the knowledge gap, our study sought to understand the renal response to SEB shock, focusing on the interaction of SEB with CD1d in RPTECs.

\section{Methods \\ Cells and reagents}

Human RPTECs were purchased from Lonza (Frederick, MD). SEB and biotinylated SEB (bt-SEB) (95\% pure, vendor defined) were purchased from Toxin Technology, Inc. (Sarasota, FL). CD1d-(Immunoglobin) Ig recombinant protein, which has extracellular MHC class I-like domains of the human CD1d molecule fused with the $\mathrm{V}_{\mathrm{H}}$ regions of mouse IgG1, was purchased from BD Biosciences (San Jose, CA). Horse radish peroxidase (HRP)-linked antimouse $(\mathrm{H}+\mathrm{L})$ horse antibody (HRP-anti-mouse) was obtained from Cell Signaling Technology, Inc. (Danvers, MA). Mouse IgG (msIgG), a polyclonal antibody purified from pooled mouse serum by fractionation and ionexchange chromatography, was from Axela, Inc. (Toronto, Ontario, Canada). Biotin-SP-AffiniPure Donkey AntiRabbit IgG $(\mathrm{H}+\mathrm{L})$ antibody (bt-Dar) was from Jackson ImmunoResearch Laboratories (West Grove, PA). 1Component TMB (3,3',5,5'-Tetramethylbenzidine) Membrane Peroxidase Substrate (a precipitable form of TMB) was from KPL, Inc. (Gaithersburg, MD). All other antibodies used in this study were purchased from Abcam, Inc., Cambridge, MA.

\section{Diffraction-based dotReady ${ }^{\mathrm{TM}}$ immunoassay}

The assays were performed in biosensors with avidin surface chemistry (Axela, Inc.). All experiments were carried out with the dotLab ${ }^{\oplus}$ System (Axela, Inc.).

\section{Principle of dot $^{\circledR}$}

The $\operatorname{dot}^{\oplus}$ (diffraction optics technology) utilizes diffraction grating to analyze real-time protein-protein interactions. A capture reagent is immobilized on a specific pattern of lines on the surface of a prism-shaped dotLab ${ }^{\oplus}$ Sensor. The sensor surface forms the base of a $10 \mu \mathrm{L}$ flow cell. A series of discrete diffraction beams are generated when illuminating the underside of each assay spot with a laser. The subsequent capture of the binding partner alone or bound to a detector antibody increases the average height of the surface pattern and causes an increase in the diffraction intensity (DI) signal that is recorded in real-time. The DI signal is directly related to the size and quantity of the bound complex $[42,43]$. The size of the bound complex is not limited to the addition of proteins, as previous studies [44] have shown that oxidization of TMB, a precipitable form of HRP substrate, can cause specific and localized precipitations on the sensor surface and result in a significant increase of DI signals. 
The principle mechanism governing the assay performance is very similar to the other industry standards such as the BIAcore with a primary difference in their tethered ligand characters [45]. Many studies reported comparable performance of $\operatorname{dot}^{\circ}$ technology in comparing other platforms such as BIAcore, amperometric assay or other methods for detecting real-time bimolecular interactions [46-48].

\section{dotReady ${ }^{\circledast}$ assay}

An avidin sensor was washed with running buffer containing PBS $(0.154 \mathrm{M} \mathrm{NaCl}, 0.01 \mathrm{M}$ phosphate, $\mathrm{pH} 7.4)$ with $0.025 \%(\mathrm{v} / \mathrm{v})$ Tween-20, and the surface was blocked to minimize nonspecific binding using bovine serum albumin (BSA) blocking buffer ( $5 \mathrm{mg} / \mathrm{ml}$ of BSA in running buffer) for $5 \mathrm{~m}$ in a mixing mode that repeatedly reverses the flow directions within the sensor. This mode was used in all subsequent incubations unless noted otherwise. Subsequently, the sensor was incubated with $10 \mu \mathrm{g} / \mathrm{ml}$ of bt-SEB for $10 \mathrm{~m}$ and washed with running buffer. BSA blocking buffer was applied and incubated for $5 \mathrm{~m}$. The dimer of CD1d:Ig at 1 $\mu \mathrm{g} / \mathrm{ml}$ was added and incubated for the next $10 \mathrm{~m}$. The sensor was washed with running buffer prior to a $5 \mathrm{~m}$ incubation of the BSA blocking buffer. HRP-anti-mouse horse antibody at a concentration of $1 \mu \mathrm{g} / \mathrm{ml}$ was added to the sensor and incubated for $10 \mathrm{~m}$. The sensor was washed with running buffer and PBS buffer. Finally, TMB was introduced and incubated for $10 \mathrm{~m}$ in static mode (i.e., the flow stopped) and DI was reported (Figure 1A).
The change in DI was recorded during each loading episode. Prior to the introduction of each reagent, the sensor was blocked with the BSA blocking buffer to establish the baseline. The delta $(\Delta)$ (the change of DI signals from the introduction of one reagent to the next one) indicated the extent of binding during each step. Each binding event was independent from each other and evaluated separately. The DI signals derived from each step of sequential addition were normalized against the delta of bt-SEB binding (as "normalized DI"), thus minimizing inter-sensor variations. Such ratio comparison is typical and has been reported previously $[48,49]$.

The specificity of CD1d in binding SEB was evaluated by systematically replacing bt-SEB with bt-Dar (Additional file 1: Figure S1A) and CD1d:Ig with BSA (Additional file 2: Figure S1B), keeping the rest of the assay protocol unchanged. Furthermore, the efficacy of CD1d:Ig fusion protein was validated with two separate experiments. In one of them, the Ig tag uncoupled from CD1d was presented instead of CD1d:Ig fusion protein, keeping the rest of the protocol unaltered. In the second experiment, HRP was presented coupled with nonspecific mouse antibody, instead of the CD1d-specific mouse antibody. The normalized DIs reported from each of the scenarios were presented along with the DI derived from the standard assay protocol described above (Additional file 3: Figure S1C)

The assay was repeated with a range of concentrations of CD1d:Ig fusion protein $(0,0.05,0.1,0.5$, and $1.0 \mu \mathrm{g} / \mathrm{ml})$, keeping the rest of the assay conditions unchanged, and
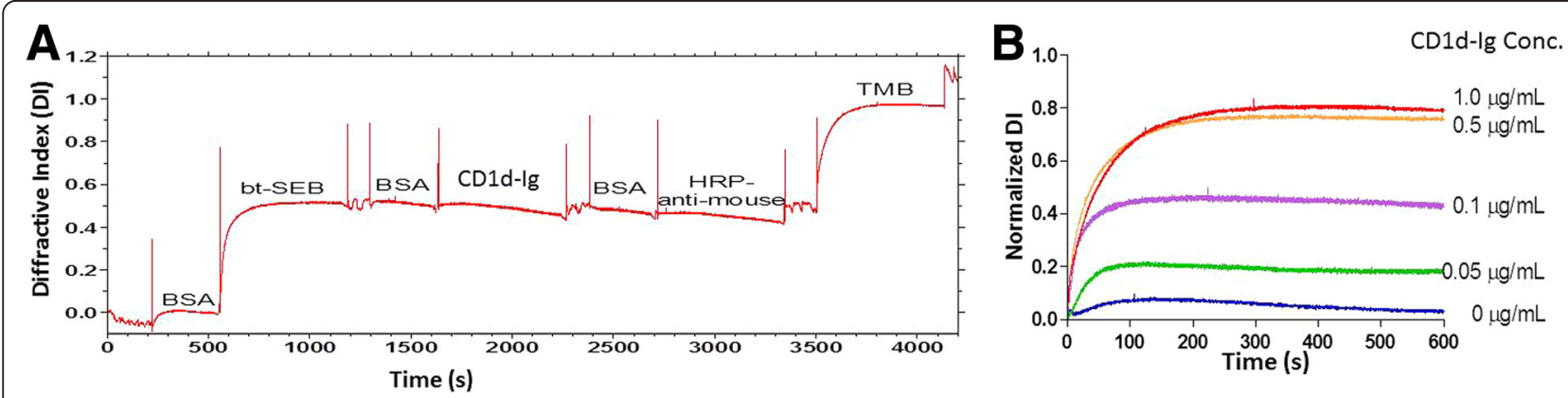

Figure 1 The kinetics and specificity of the capture of CD1d with biotinylated SEB (bt-SEB) with the dotReady system were characterized.

A. Kinetics of SEB-CD1d interaction: A representative real-time trace depicts the kinetics of capturing CD1d with biotinylated SEB (bt-SEB) on the dotReady ${ }^{\mathrm{TM}}$ system. Here, the X-axis indicates the assay duration in seconds (s) and the Y-axis indicates measured diffractive index (DI). $10 \mu \mathrm{g} / \mathrm{ml}$ bt-SEB was immobilized on the avidin sensor during a $10 \mathrm{~m}$ incubation. CD1d:Ig fusion protein (extracellular major histocompatibility complex (MHC) class I-like domains of human $C D 1 d$ fused with $V_{H}$ regions of mouse $\left.\mathrm{lgG} 1\right)(1 \mu \mathrm{g} / \mathrm{ml})$ was added and incubated for a $10 \mathrm{~m}$ post-BSA treatment. After a second round of BSA washing, the complex was incubated with horse anti-mouse HRP-conjugated secondary antibody (HRP-anti-mouse; 1 $\mu \mathrm{g} / \mathrm{ml})$ for another $10 \mathrm{~m}$. Finally, TMB was added as the reporting agent. The shift of DI with time in seconds is presented herein. For all DI tracing, the upward spikes are air gaps separating reagents. All non-labeled portions are attributed to the wash steps. Increased DI signals showing as an upward ramp indicated the binding of a reagent in this step. The highest ramp after TMB presentation suggests the successful binding of SEB and CD1d. B. Signal increases with increasing concentrations of CD1d presented to bind SEB: The assay followed the same sequential addition described in Figure 1A, namely, the presentation of biotinylated SEB to avidin sensor, followed by addition of CD1d:Ig fusion protein, horse anti-mouse HRP-conjugated secondary protein and TMB, respectively intercepted with BSA washing. The normalized DI signals measured during $600 \mathrm{~s}$ after the introduction of TMB were plotted against the serially increasing concentrations of CD1d from 0 to $1.0 \mu \mathrm{g} / \mathrm{ml}$. A sigmoidal dose-response (variable slope) function was used to fit the curve, $R^{2}=0.9984$. The positive correlation of the increased concentration of CD1d:Ig with enhanced DI while keeping the concentration of the other reagents constant validated the assay specificity. 
the corresponding DIs for each CD1d:Ig concentration were reported in Figure 1B. All data recorded in dotLab ${ }^{\circ}$ Software were analyzed using GraphPad Prism ${ }^{\circ}$ (GraphPad Software, Inc., San Diego, CA).

\section{RPTEC culture and fluorescence-based reporting}

RPTECs were grown in REBM culture medium supplemented with a bullet kit containing hEGF, hydrocortisone, epinephrine, insulin, triiodothyronine, transferrin and GA-1000 (Lonza, MD). Cells were cultured and passaged as per supplier's protocol using recommended reagents. Cells at passage 5-6 were plated in chamber slides at $1 \times 10^{6}$ cells $/ \mathrm{ml}$ for $24 \mathrm{~h}$ at $37^{\circ} \mathrm{C}$. SEB was added to the cells at a concentration of $100 \mu \mathrm{g} / \mathrm{ml}$ and incubated at $37^{\circ} \mathrm{C}$ for $15 \mathrm{~m}, 30 \mathrm{~m}, 1 \mathrm{~h}$ and $2 \mathrm{~h}$. Postincubation, the cells were washed twice with $1 \times$ PBS. For the CD1d reporting assays (Figure 2), the cells were treated with mouse anti-CD1d antibody, incubated for $30 \mathrm{~m}$ on ice and washed with $1 \times$ PBS. Subsequently, $5 \mu \mathrm{l}$ of Alexa 594-conjugated goat anti-mouse antibodies in $1 \times$ PBS was added and incubated for $5 \mathrm{~m}$ on ice and washed. The cells were resuspended in $1 \mathrm{ml}$ of formaldehyde and 1× PBS mix (1:1) for $15 \mathrm{~m}$ at room temperature, washed twice using $1 \times$ PBS, subsequently resuspended in $1 \mathrm{ml}$ of $1 \times \mathrm{PBS}$, and imaged using a fluorescent microscope equipped with a digital camera (Olympus Optical Company, Melville, NY). RPTECs without SEB exposure were processed similarly and imaged, and Figure 2 reports surface expression of CD1d after $1 \mathrm{~h}$ exposure to SEB or PBS treatment (negative control).

For the SEB reporting assays (data not shown), the cells were treated with rabbit anti-SEB antibody for 30 $m$ on ice, washed with $1 \times$ PBS, and incubated on ice
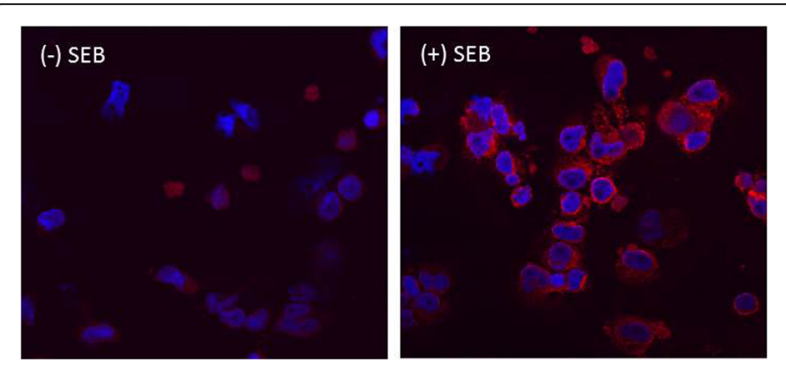

Figure 2 Influence of SEB on CD1d cell surface expression: Fluorescence-based CD1d reporting: 1 × $10^{6}$ cells $/ \mathrm{ml} \mathrm{RPTECs}$ were exposed to $100 \mu \mathrm{g} / \mathrm{ml} \mathrm{SEB}$ for $1 \mathrm{~h}$, washed and then probed using the mouse anti-CD1d antibody coupled to Alexa 594-conjugated goat anti-mouse (Red spots). This image is designated as (+) SEB. The (-) SEB image was obtained from the cells treated with the same method; however, SEB was replaced with PBS. All images are at 60X magnification captured with a fluorescent microscope equipped with a digital camera (Olympus Optical Company). The red spots represent the CD1d expressed on the cell surface. with FITC-conjugated goat anti-rabbit antibodies for 5 $\mathrm{m}$. Subsequently, the cells were washed and resuspended in $1 \mathrm{ml}$ of formaldehyde and PBS mix (1:1). After incubation at room temperature for $15 \mathrm{~m}$, it was washed with $1 \times$ PBS, resuspended again in $1 \times$ PBS and proceeded to imaging. The images were analyzed using a fluorescent microscope equipped with a digital camera (Olympus Optical Company). The control chamber slide had no SEB loading.

For dual reporting, RPTECs were harvested, plated in chamber slides and treated with SEB for increasing durations $(15 \mathrm{~m}, 30 \mathrm{~m}, 1 \mathrm{~h}$ and $2 \mathrm{~h})$, as per the protocol described earlier. Post-treatment, the cells were incubated on ice with mouse anti-CD1d antibody; after $30 \mathrm{~m}$ incubation, rabbit anti-SEB antibody suspended in PBS was added and the incubation on ice was continued for another $30 \mathrm{~m}$. The cells were washed twice, and the Alexa Fluor 594-conjugated goat anti-mouse antibody and FITC-conjugated goat anti-rabbit antibody suspended in $1 \mathrm{x}$ PBS were added to incubate for $5 \mathrm{~m}$. Subsequently, the cells were washed, resuspended in $1 \mathrm{ml}$ of formaldehyde and PBS mix (1:1). After incubating at room temperature for $15 \mathrm{~m}$, it was washed with $1 \times \mathrm{PBS}$, resuspended again in $1 \times$ PBS and proceeded to imaging. The images were analyzed using a fluorescent microscope equipped with a digital camera (Olympus Optical Company). Figure 3 shows the fluorescence images of $15 \mathrm{~m}, 30 \mathrm{~m}, 1 \mathrm{~h}$ and $2 \mathrm{~h}$ of SEB treatments.

\section{Immunoblotting to report time dependent binding efficiency of SEB to CD1d}

Co-immunoprecipitation of CD1d-bound SEB was carried out using the Universal Magnetic Co-IP kit (Active-Motif, Carlsbad, CA) according to the manufacturer's instructions. Briefly, RPTECs were exposed to SEB for $1 \mathrm{~h}$ and 2 $\mathrm{h}$ as per the protocol described earlier. An aliquot of 400 ng of whole cell extract and $2 \mu \mathrm{g}$ of mouse anti-CD1d antibody was combined in a pre-chilled $1.5 \mathrm{ml}$ tube and incubated for $2 \mathrm{~h}$ at $4^{\circ} \mathrm{C}$ on a rolling shaker. Samples were centrifuged for $30 \mathrm{~s}$ at $4000 \mathrm{rpm}$ at $4{ }^{\circ} \mathrm{C}$, then $25 \mu \mathrm{l}$ of Protein G Magnetic Beads was added to each tube. The mixture was incubated for $1 \mathrm{~h}$ at $4^{\circ} \mathrm{C}$ and centrifuged for $30 \mathrm{~s}$ at $4000 \mathrm{rpm}$ at $4^{\circ} \mathrm{C}$. Each tube was placed on a magnetic stand to pellet the beads, supernatants were discarded, and the pellets were resuspended and subsequently washed with $1 \times$ PBS. Immunoblotting was performed according to the protocol described elsewhere (Additional file 4: Figure S2) [50].

\section{qPCR assay to assess CD1d expression}

The protocol described earlier was followed to harvest RPTECs and to expose them to $100 \mu \mathrm{g} / \mathrm{ml}$ SEB for $1 \mathrm{~h}$, $2 \mathrm{~h}$ and $24 \mathrm{~h}$. Total RNA was isolated from RPTECs in a $125 \mathrm{ml}$ flask using TRIzol reagent (Invitrogen, Carlsbad, 


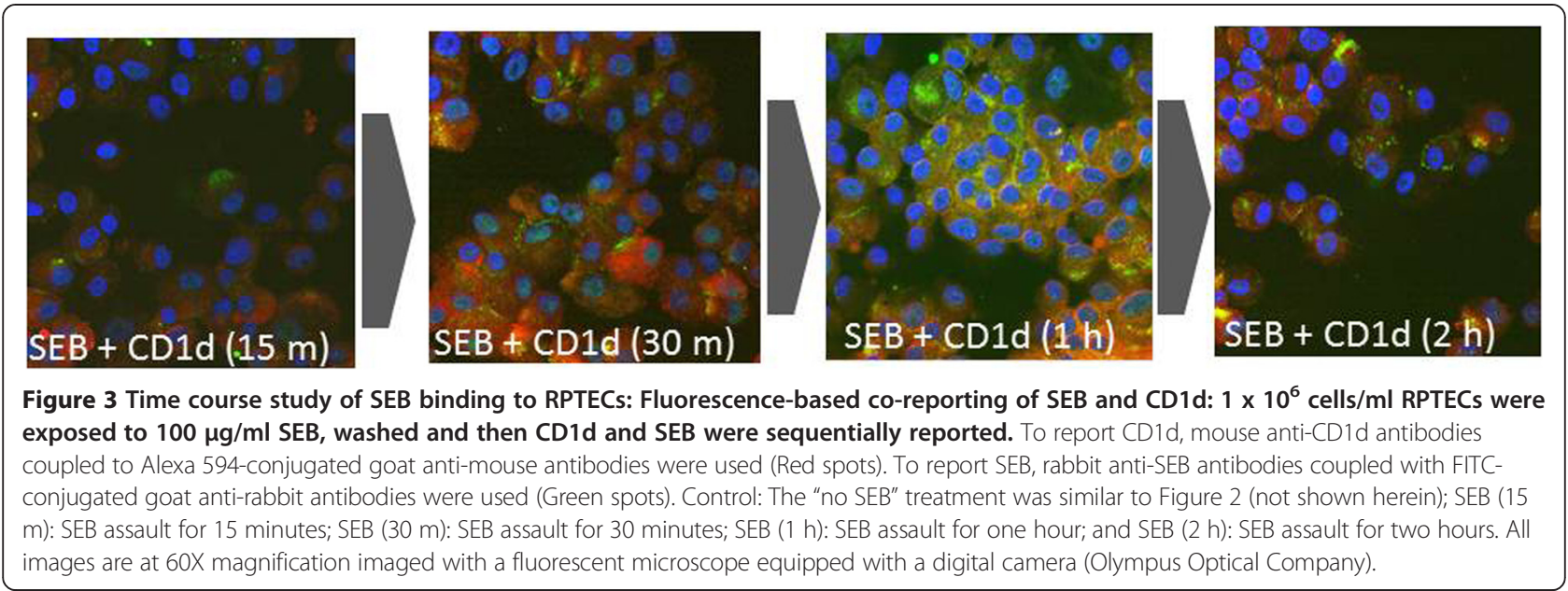

CA) as per manufacturer's protocol. RNA was quantified via spectrophotometry followed by analysis with a Bioanalyzer 2100 (Agilent Technologies, Santa Clara, CA).

The primer sequences used for the amplification of CD1d were 5'-GGGCACTCAGCCAGGGGACATCCTG CCCAA-3' as forward and 5'-GATACAAGTTTGCACA CCTTTGCACTTCTG-3' as reverse. The specificity of each primer sequence was further confirmed by running a BLAST search. Reverse transcription and real-time PCR reactions were carried out using iScript cDNA Synthesis Kit (Bio-Rad, Hercules, CA) and Real-time PCR kit (Roche, Indianapolis, IN), respectively. Five technical replicates of each reaction were completed in an I-Cycler machine (Bio-Rad). Each sample was also amplified using a primer pair targeting $18 \mathrm{~S}$ ribosomal RNA as the housekeeping gene, whose selection for the present purpose was instituted because of many past observations [51-53]. The resultant cycle threshold data from each real-time PCR experiment was converted to fold-change by using an established algorithm [54]. The fold-change results obtained at three time points were compared by Welch's corrected $t$-test $p$ values * $<0.05$ (Figure 4).

\section{Immunological inhibition of CD1d fluorescence-based reporting}

RPTECs were harvested as per the protocol described earlier, and $1 \times 10^{6}$ cells $/ \mathrm{ml}$ loaded in chamber slides were treated with $1 \mu \mathrm{g} / \mathrm{ml}$ rabbit anti-CD1d antibody (or PBS as negative control), termed as SEB + CD1d in Figure 5 , for $30 \mathrm{~m}$. Post-wash, the cells were exposed to $100 \mu \mathrm{g} / \mathrm{ml}$ SEB for $1 \mathrm{~h}$. There was one additional set of controls termed as inhCD1d (Figure 5) that did not undergo the SEB treatment, but the rest of the protocol was carried out as previously described. Post-treatment, the cells were incubated on ice with anti-SEB rabbit antibody for $30 \mathrm{~m}$ on ice. The cells were washed twice, and the goat anti-rabbit FITC-conjugated secondary antibody suspended in 1× PBS was added and incubated for $5 \mathrm{~m}$. Subsequently, the cells were washed, and resuspended in $1 \mathrm{ml}$ of formaldehyde and PBS mix (1:1). After incubating at room temperature for $15 \mathrm{~m}$, it was washed with $1 \times$ PBS and resuspended again in $1 \times$ PBS as before. The fluorescence intensity was estimated using the FX scanner (BioRad). A "no cell" control was used as the baseline, and their average read outs were used for normalization purpose. (Treated - Control $\pm \mathrm{SE})(N=5)$. Welch's corrected $t$-test $p$ values * $<0.05$ was used for all statistical notations.

\section{Results}

\section{Binding efficiency and specificity of SEB to CD1d}

The dotLab ${ }^{\circ}$ System with diffractive optics technology $\left(\operatorname{dot}^{\circ}\right)$ was used to systematically investigate the binding specificity of SEB to CD1d. In this study, biotinylated

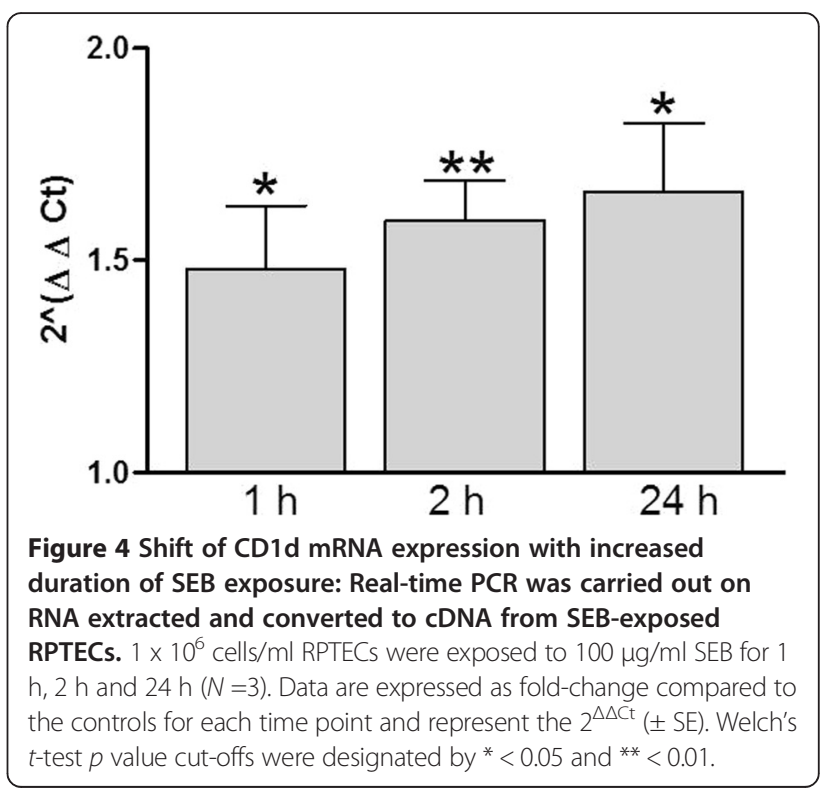




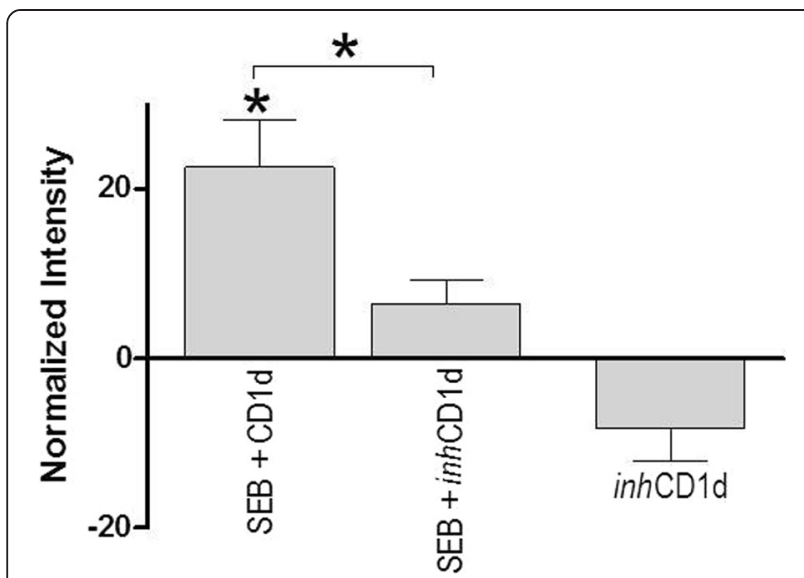

Figure 5 Inhibition of CD1d impedes SEB binding to RPTECs: $1 \times 10^{6}$ cells $/ \mathrm{ml}$ RPTECs were treated with mouse anti-CD1d antibody $(1 \mu \mathrm{l} / \mathrm{ml})$ or PBS for $30 \mathrm{~m}$; post-wash, the cells were exposed to $100 \mu \mathrm{g} / \mathrm{ml}$ SEB for $1 \mathrm{~h}$. Anti-SEB rabbit antibody followed by goat anti-rabbit FITC-conjugated secondary antibody was used for reporting purposes. A "no cell" control was used as baseline and the average was used to normalize intensity (Treated - Control \pm $\mathrm{SE})(N=5)$; Welch's $t$-test $p$ value cut-offs were designated by * $<0.05$. The first bar (designated as SEB + CD1d) shows SEB-CD1d binding in the absence of the inhibitory anti-CD1d antibody. A significant reduction of signal was observed in presence of the inhibitory antiCD1d antibody (second bar; designated as SEB + inhCD1d). Third bar plot (inhCD1d) shows the specificity of the anti-SEB primary antibody and the corresponding secondary antibody towards the anti-CD1d antibody in the absence of SEB exposure.

SEB (bt-SEB) was immobilized on a sensor equipped with avidin surface chemistry. The binding as the result of avidin-biotin interaction was displayed as an elevated upward ramp of DI at the end of around $500 \mathrm{~s}$ episode of BSA incubation (Figure 1A). Sequential BSA washing and the loading of CD1d:Ig to bt-SEB (each episode of incubation lasted for approximately 500 s) did not register the detectable increment of DI. The conjugation of SEB and CD1d took place nonetheless, as demonstrated by presenting an HRP-linked detector antibody (HRPanti-mouse) that was conjugated to the Ig domain of CD1d:Ig duplex. Subsequently, DI was ramped as we introduced TMB, a precipitating substrate of HRP.

Four negative control assays were carried out to test the specificity of the conjugation between SEB and CD1d. In a mock surface control, nonspecific antibody bt-Dar was immobilized on the surface. CD1d:Ig followed by HRPlinked anti-mouse antibody was sequentially introduced as described earlier. TMB administration however, failed to increase the DI signals (Additional file 1: Figure S1A) denying the chance of nonspecific binding of CD1d in this assay. In an analyte blank control, BSA blank instead of CD1d:Ig was introduced on the immobilized SEB surface. Following the same assay sequence, weak signal amplification was recorded after TMB loading (Additional file 2: Figure S1B). Henceforth the specificity of the HRPlinked antibodies to CD1d:Ig was established.

Furthermore, we repeated this experiment, replacing CD1d:Ig with either (i) the Ig tag uncoupled from CD1d or (ii) HRP linked to nonspecific mouse antibody (HRP-1A11), which can only bind to human cardiac troponin T (Additional file 3: Figure S1C). Presentation of Ig-only substrate feebly increased DI while HRP-1A11 failed to increase DI after $600 \mathrm{~s}$ incubation.

The assay was further repeated with serially increasing concentrations of CD1d:Ig from $0.05 \mu \mathrm{g} / \mathrm{ml}$ to $1.0 \mu \mathrm{g} / \mathrm{ml}$ (Figure 1B), showing concurrent increment of the corresponding fluorescence signals.

\section{Longitudinal dynamics of the conjugation of SEB and CD1d on RPTECs' surface}

The longitudinal dynamics of RPTECs' response to SEB exposures were evaluated by incubating the cells with SEB for increasing durations. To examine whether SEB treatment can affect the intracellular trafficking of CD1d, the distribution of CD1d expression on the RPTEC's surface was monitored with and without SEB treatment. Compared to the untreated cells, the $1 \mathrm{~h} \mathrm{SEB}$ treatment elevated CD1d expression on the RPTEC's surface as reported by the Alexa 594-based fluorescence assay (Figure 2).

The outcome was further validated by the qPCR assay of the expression of CD1d after increased durations of SEB exposures. After $1 \mathrm{~h}$ SEB treatment, CD1d expression became significantly higher $(p<0.05)$ than that of the untreated control samples. Longer durations of SEB treatment retained the elevated level of CD1d mRNA transcriptomic expression (Figure 4).

The longitudinal dynamics of SEB binding to RPTEC were reported with accompanying CD1d expression on the cell surface (Figure 3). Sequential reporting of CD1d and SEB using antibodies with mutually exclusive binding epitopes showed co-localization of SEB and CD1d on the cell surface. After displaying a gradual increment until $1 \mathrm{~h}$, the expression density gradually declined as reported after $2 \mathrm{~h}$ SEB exposure (Figure 3).

The reduction of $\mathrm{SEB}$ concentration conjugated to CD1d after $2 \mathrm{~h}$ incubation was verified with an immunoblotting study (Figure S2). Here, post- 1 h and -2 h SEB exposure, the CD1d protein was surface-captured and SEB levels were reported by sequential addition of antibodies with mutually exclusive capturing epitopes. The concentration of SEB conjugated to CD1d was found diminished after $2 \mathrm{~h}$ SEB exposure.

\section{Consequences of inhibiting CD1d of RPTECs before SEB exposure}

RPTECs were pre-treated with polyclonal anti-CD1d antibody before regular SEB treatment. The SEB 
reporting profile demonstrated the consequences of inhibiting CD1d from conjugating with the antigens. In Figure 5, the first bar from the left $(\mathrm{SEB}+\mathrm{CD} 1 \mathrm{~d})$ displayed the intensity of SEB conjugated to CD1d in the absence of the anti-CD1d antibody. A significant reduction of signal was observed in presence of the anti-CD1d antibody (second bar plot; SEB + inhCD1d) that inhibited the CD1d ability to conjugate SEB. A faint signal was nevertheless recorded. The third bar showed a negative signal (inhCD1d), which potentially attested that the anti-SEB primary antibody (and the corresponding secondary antibody) shared no common epitope with antiCD1d polyclonal antibody.

\section{Discussion}

Multiple studies reported the critical protective role played by the kidney in excretion of SEB [20-26]. RPTECs selectively express CD1d, which play a significant (and MHC II-independent) role in antigen sensing and recognition process [13,29-31]. The present study sought to elucidate the renal pathogenesis of SEB with the primary focus on the toxin's interaction with CD1d expressed on RPTECs.

Time sensitive co-localization of SEB with CD1d expressed on RPTECs' surface (Figure 3) was found in accordance with others' findings $[12,32,33,55]$. The renal localization of SEB began as the toxin conjugated to CD1d; in fact, the co-localization, which began merely $15 \mathrm{~m}$ post-exposure possibly explained the etiology behind the 'jumpstart' of host immunity after CD1d-NKT cell conjugation [35].

We also showed that SEB conjugated directly to CD1d using the $\operatorname{dot}^{\circ}$ platform (Figure 1A), a technology enabling us to probe the protein-ligand interaction in a real-time format with proven success at per the other industry standards such as BIAcore $[47,48]$. The direct and specific binding of renal CD1d with SEB was further attested by the selective immunoinhibition of CD1d (Figure 5) that inhibited SEB to bind RPTECs at all. This information is rather important in context to the evidence that showed no direct involvement of CD1d in tuberculosis antigen presentation [55,56] and to other reports suggesting non-conventional sAg presenting sites $[18,19]$.

We paid particular attention to minimize the risk of false positive result of $\operatorname{dot}^{\bullet}$ assay by conducting an array of validation experiments. These included the probing of (i) the blank capturing agents on the surface that attested the specificity of the SEB and CD1d binding (Additional file 1: Figure S1A). (ii) The analyte blank control for CD1d attested the specificity of the HRP-linked antibody to CD1d:Ig (Additional file 2: Figure S1B). (iii) The analyte quantity control investigated the dose titration of the CD1d amount and verified the stoichiometric dynamics between CD1d and SEB (Figure 1B). (iv)The analyte tag negative control replacing the Ig tag control of CD1d with msIgG reconfirmed the specificity of Ig to HRP-like antibody (Additional file 3: Figure S1C). Finally (v) the HRPlinked detector antibody was replaced by HRP-1A11 as a nonspecific detector for CD1d that revalidated the same (Additional file 3: Figure S1C). Together these assay results imparted maximum confidence on the present inference about the direct and specific conjugation of SEB to CD1d.

Present methodology, however was not able to empirically measure the affinity between SEB and CD1d. This could be due to a weak SEB-CD1d interaction, which was potentially unlikely in light of the past studies that demonstrated high affinity of CD1d for glycolipids [57]; in fact, our data displaying a short duration required to form SEB-CD1d conjugates further supports this report [57]. As a more probable alternative, the requirement of a signal enhancing addendum (TMB, in this case) could be simply due to the fact that the reagents and the experimental conditions used in vitro in dot $^{\circ}$ experiments were different from what happens in the cell. A possible role of a secondary adjunct facilitating SEB CD1d conjugation in the cellular environment could not be ruled out; a more comprehensive study is required.

The fluorescence images obtained from RPTECs displayed the density of SEB conjugated with CD1d without the additional help from signal amplification agents. The result indicated that the SEB exposure prompted the elevation of CD1d expression on the cell surface (Figure 2).

As shown in Figure 3, the co-localization profile of SEB and CD1d displayed a gradual increment of their densities up to $1 \mathrm{~h}$ and concluded with a subsequent decline, which suggested the eventual trafficking of SEB. Also, Figure 2 suggested that the SEB challenges prompted CD1d expression on the cell surface after $1 \mathrm{~h}$ incubation, while the immunoblotting result (Additional file 4: Figure S2) validated the potential excretion of SEB. The enrichment of the cell-bound SEB depleted as the incubation period was extended to second hour. The present study was limited by not tracking the unbound SEB during the second hour of exposure.

Interestingly, the transcriptomic expression of CD1d remained elevated (Figure 4), possibly long after the SEB was sequestered. Of note, a previous study demonstrated a long half-life of CD1d [58]. These outcomes, coupled with Figure 2 displaying the SEB-induced the cell surface expression of CD1d may indicate that SEB regulates CD1d expression on RPTEC's surface. Further investigation of CD1d in RPTECs could have major clinical interest, particularly in the context of understanding a robust system that enables facilitation of a delayed antigenic cleansing program [59]. 
The essential role of renal CD1d in SEB-associated pathogenesis was further validated with a fluorescencebased assay demonstrating the sequential inhibition of CD1d and preventing its binding to SEB (Figure 5). In agreement, multiple studies observed diminished host defense as a result of the targeted CD1d deficiency $[37,40,59,60]$. It was, however, beyond the scope of the present study to suggest putative SEB-induced toxemia through CD1d-independent routes. Such possibilities, nonetheless, have been suggested by the recruitment of CD1d-restricted T-cells [40,41] and invariant Natural Killer cells [15].

\section{Conclusion}

In conclusion, the present study examined the renal pathogenesis of SEB, which revealed a potential functional role for CD1d in antigen recognition, cleansing and sequestration $[12,22,26,27]$. The binding specificity of CD1d and SEB was established. A rapid conjugation of CD1d with SEB implicated a possible high affinity between the two molecules, particularly in RPTECs. Such prompt interaction may facilitate the rapid surge of cytokines triggered by the crosstalk between CD1d and NKT cells [35].

The display of the co-localization of SEB and CD1d at the RPTEC's surface and the decline of the SEB population on the cell surface after CD1d immunoinhibition suggested that RPTECs undertake an exclusive CD1dmediated antigen presentation process. The transcriptomic elevation (and cell surface expression) of CD1d in RPTECs was concurrent with SEB exposure, which remained elevated potentially long after SEB trafficking. Together, a feedback-controlled conjugation of SEB and CD1d is suggested in carrying out the recognition, cleansing and sequestration of SEB. Further investigation is required to establish this CD1d-mediated mechanism as a potential downstream mechanism of SEB excretion and a prospective therapeutic target.

\section{Additional files}

Additional file 1: Figure S1A. Validation of the kinetics of SEB-CD1d interaction: Mock surface control: nonspecific surface antibody (bt-Dar) did not bind to CD1d: As a mock surface control for bt-SEB, nonspecific antibody bt-Dar (identified by the dashed circle) was immobilized on the surface. TMB loading failed to increase the DI signals, indicating that there wasn't nonspecific binding of CD1d to bt-Dar.

Additional file 2: Figure S1B. Validation of the kinetics of SEB-CD1d interaction: Analyte control, BSA blank replacement of CD1d failed to emit signal: No CD1d:Ig fusion protein was presented in the assay, instead, a blank BSA wash was performed. The changed reagent is identified by the dashed circle. The specificity of horse anti-mouse HRP-conjugated antibody to CD1d:Ig fusion protein was reported with no increment of DI trace after TMB presentation.

Additional file 3: Figure S1C. Validation of the kinetics of SEB-CD1d interaction: Analyte tag control: Signal decreased as (a) the lg tag uncoupled from CD1d was presented: The normalized DI signal during $600 \mathrm{~s}$ after the introduction of TMB was plotted against the concentrations of CD1d from $1.0 \mu \mathrm{g} / \mathrm{ml}$ as reported in Additional file 1: Figure S1A (red line). A polyclonal anti-mouse antibody (mslgG, a mixture containing all subclass of IgG including IgG1) was presented as an analyte-negative. The rest of the assay was same as described in Additional file 1: Figure S1A. The Ig tag without CD1d failed to signal as reported in the blue line (mslgG (a)). The signal decreased as (b) HRP conjugated with nonspecific antibody was presented: HRP-1A11, a HRP-linked nonspecific mouse antibody (only capable of binding human cardiac troponin T), was employed as a HRP detector negative control. The rest of the assay was the same as described in Additional file 1: Figure S1A. The nonspecific mouse antibody failed to couple with CD1d:Ig fused protein, reporting no signal as indicated in the green line (HRP-1A11 (b)).

Additional file 4: Figure S2. Time course study of SEB binding to RPTECs: Immunoblotting to assess SEB levels by measuring bound CD1d: $1 \times 10^{6}$ cells/ ml RPTECs were exposed to $100 \mu \mathrm{g} / \mathrm{ml}$ SEB for two time durations, namely $1 \mathrm{~h}$ and $2 \mathrm{~h}$; controls were not exposed to SEB. Cells were coupled with mouse anti-CD1d antibody and immunoprecipitated with protein $\mathrm{G}$ magnetic beads. Immunoblotting was completed by presenting the conjugate to anti-SEB rabbit antibody followed by goat anti-rabbit HRP conjugated secondary antibody.

\section{Competing interests}

The authors declare that they have no competing interests. YL and SM have no present financial interest associated with Axela, Inc.

\section{Authors' contributions}

MJ, JWS, SM and RH conceived and designed the assay. S-AM and YL carried out the experiment. NC, SM and $\mathrm{RH}$ analyzed the data. NC and $\mathrm{RH}$ prepared the manuscript. All authors read and approved the document.

\section{Acknowledgements}

Authors like to acknowledge the insightful contribution from the internal editor Dr. Julia Scheerer. RH, NC, SM and MJ like to acknowledge the funding from DTRA: CB3293-Host Factors and Shock-Inducing Bioagents.

\section{Disclaimer}

The views, opinions, and/or findings contained in this report are those of the authors and should not be construed as official Department of the Army position, policy, or decision, unless so designated by other official documentation.

Citations of commercial organizations or trade names in this report do not constitute an official Department of the Army endorsement or approval of the products or services of these organizations.

\section{Author details}

'Integrative Systems Biology, US Army Center for Environmental Health Research Fort Detrick, 568 Doughten Drive, Fort Detrick, MD 21702-5010, USA. ${ }^{2}$ Axela, Inc., 50 Ronason Drive, Suite 105, Toronto, ON M9W 1B3, Canada. ${ }^{3}$ The Burn Center, Department of Surgery, Washington Hospital Center, Washington, DC 20010, USA.

Received: 17 December 2014 Accepted: 13 January 2015 Published online: 04 February 2015

\section{References}

1. Balaban N, Rasooly A. Staphylococcal enterotoxins. Int J Food Microbiol. 2000;61(1):1-10.

2. Florquin S, Aaldering L. Superantigens: a tool to gain new insight into cellular immunity. Res Immunol. 1997;148(6):373-86.

3. Krakauer T. Therapeutic down-modulators of staphylococcal superantigeninduced inflammation and toxic shock. Toxins. 2010;2(8):1963-83.

4. Eriksson BK, Andersson J, Holm SE, Norgren M. Invasive group A streptococcal infections: T1M1 isolates expressing pyrogenic exotoxins A and $B$ in combination with selective lack of toxin-neutralizing antibodies are associated with increased risk of streptococcal toxic shock syndrome. J Infect Dis. 1999;180(2):410-8.

5. Le Loir Y, Baron F, Gautier M. Staphylococcus aureus and food poisoning. GMR. 2003;2(1):63-76. 
6. Henghold 2nd WB. Other biologic toxin bioweapons: ricin, staphylococcal enterotoxin B, and trichothecene mycotoxins. Dermatol Clin. 2004;22 (3):257-62. v.

7. Larkin EA, Stiles BG, Ulrich RG. Inhibition of toxic shock by human monoclonal antibodies against staphylococcal enterotoxin B. PLoS One. 2010;5(10):e13253.

8. Farag N, Mahran L, Abou-Aisha K, El-Azizi M. Assessment of the efficacy of polyclonal intravenous immunoglobulin $\mathrm{G}(\mathrm{IVIG})$ against the infectivity of clinical isolates of methicillin-resistant Staphylococcus aureus (MRSA) in vitro and in vivo. Eur J Clin Microbiol Infect Dis. 2013;32(9):1149-60.

9. Eichacker PQ, Parent C, Kalil A, Esposito C, Cui X, Banks SM, et al. Risk and the efficacy of antiinflammatory agents: retrospective and confirmatory studies of sepsis. Am J Respir Crit Care Med. 2002;166(9):1197-205.

10. Tunkel AR. Septic shock and toxic shock: Do adjunctive therapies improve outcome? Curr Infect Dis Rep. 1999;1(3):215-7.

11. Ferry $T$, Thomas $D$, Genestier AL, Bes M, Lina G, Vandenesch F, et al. Comparative prevalence of superantigen genes in Staphylococcus aureus isolates causing sepsis with and without septic shock. Clin Infect Dis. 2005;41(6):771-7.

12. Ferreyra GA, Elinoff JM, Demirkale CY, Starost MF, Buckley M, Munson PJ, et al. Late multiple organ surge in interferon-regulated target genes characterizes staphylococcal enterotoxin B lethality. PLoS One. 2014;9(2):e88756.

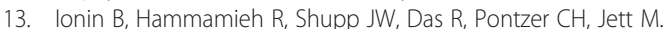
Staphylococcal enterotoxin B causes differential expression of Rnd3 and RhoA in renal proximal tubule epithelial cells while inducing actin stress fiber assembly and apoptosis. Microb Pathog. 2008;45(5-6):303-9.

14. Teixeira FM, Fernandes BF, Rezende AB, Machado RR, Alves CC, Perobelli SM, et al. Staphylococcus aureus infection after splenectomy and splenic autotransplantation in BALB/c mice. Clin Exp Immunol. 2008;154(2):255-63.

15. Rieder SA, Nagarkatti P, Nagarkatti M. CD1d-independent activation of invariant natural killer T cells by staphylococcal enterotoxin B through major histocompatibility complex class $\| / T$ cell receptor interaction results in acute lung injury. Infect Immun. 2011;79(8):3141-8.

16. Nowrouzian FL, Dauwalder O, Meugnier H, Bes M, Etienne J, Vandenesch F, et al. Adhesin and superantigen genes and the capacity of Staphylococcus aureus to colonize the infantile gut. J Infect Dis. 2011;204(5):714-21.

17. Hahn BL, Sohnle PG. Direct translocation of staphylococci from the skin surface to deep organs. Microb Pathog. 2013;63:24-9.

18. Rogers TJ, Guan L, Zhang L. Characterization of an alternative superantigen binding site expressed on a renal fibroblast cell line. Int Immunol. 1995;7 (11):1721-7.

19. Dohlsten M, Hedlund G, Segren S, Lando PA, Herrmann T, Kelly AP, et al. Human major histocompatibility complex class II-negative colon carcinoma cells present staphylococcal superantigens to cytotoxic T lymphocytes: evidence for a novel enterotoxin receptor. Eur J Immunol. 1991;21(5):1229-33.

20. Morris EL, Hodoval LF, Beisel WR. The unusual role of the kidney during intoxication of monkeys by intravenous staphylococcal enterotoxin B. J Infect Dis. 1967;117(4):273-84.

21. Hodoval LF, Morris EL, Crawley GJ, Beisel WR. Pathogenesis of lethal shock after intravenous staphylococcal enterotoxin B in monkeys. Appl Microbiol. 1968;16(2):187-92.

22. Normann SJ, Jaeger RF, Johnsey RT. Pathology of experimental enterotoxemia. The in vivo localization of staphylococcal enterotoxin B. Laboratory investigation; a journal of technical methods and pathology. 1969;20(1):17-25.

23. Staab EV, Niederhuber J, Rhoda DA, Faulkner 2nd CS, Beisel WR. Role of the kidney in staphylococcal enterotoxemia. Appl Microbiol. 1969;17(3):394-8.

24. Normann SJ. Renal fate of staphylococcal enterotoxin B. Lab Invest. 1971;25 (2):126-32.

25. Normann SJ, Stone CM. Renal lysosomal catabolism of staphylococcal enterotoxin B. Lab Invest. 1972;27(2):236-41.

26. Nagaki M, Hughes RD, Keane HM, Goka J, Williams R. Clearance and tissue distribution of staphylococcal enterotoxin $A$ in the rat and potential use of adsorbents for removal from plasma. J Med Microbiol. 1993;38(5):354-9.

27. Mino MJ, Ortiz RT, Randad P, Moffatt LT, Jordan MH, Shupp JW. Localization of superantigen virulence factors in kidney tissue of animals with Staphylococcus aureus-infected burn wounds. J Burn Care Res. 2013;34 (1):142-50.

28. Roark JH, Park SH, Jayawardena J, Kavita U, Shannon M, Bendelac A. CD1.1 expression by mouse antigen-presenting cells and marginal zone B cells. J Immunol. 1998;160(7):3121-7.
29. Shupp JW, Jett M, Pontzer $\mathrm{CH}$. Identification of a transcytosis epitope on staphylococcal enterotoxins. Infect Immun. 2002;70(4):2178-86.

30. Chatterjee $S$, Jett M. Glycosphingolipids: the putative receptor for Staphylococcus aureus enterotoxin-B in human kidney proximal tubular cells. Mol Cell Biochem. 1992;113(1):25-31.

31. Chatterjee S, Neill R, Shupp JW, Hammamieh R, lonin B, Jett M. Identification of staphylococcal enterotoxin B domains involved in binding to cultured human kidney proximal tubular cells: imparting proliferation and death. Exp Biol Med. 2007;232(9):1142-51.

32. Bendelac A, Rivera MN, Park SH, Roark JH. Mouse CD1-specific NK1 T cells: development, specificity, and function. Annu Rev Immunol. 1997;15:535-62.

33. Blumberg RS, Gerdes D, Chott A, Porcelli SA, Balk SP. Structure and function of the CD1 family of MHC-like cell surface proteins. Immunol Rev. 1995;147:5-29.

34. Porcelli SA. The CD1 family: a third lineage of antigen-presenting molecules. Adv Immunol. 1995;59:1-98.

35. Joyce S. CD1d and natural T cells: how their properties jump-start the immune system. CMLS. 2001;58(3):442-69.

36. Carnaud C, Lee D, Donnars O, Park SH, Beavis A, Koezuka Y, et al. Cutting edge: cross-talk between cells of the innate immune system: NKT cells rapidly activate NK cells. J Immunol. 1999;163(9):4647-50.

37. Ulanova M, Tarkowski A, Hahn-Zoric M, Hanson LA. Participation of CD1 molecules in the presentation of bacterial protein antigens in humans. Scand J Immunol. 1999;50(4):387-93.

38. Salamone MC, Rabinovich GA, Mendiguren AK, Salamone GV, Fainboim L. Activation-induced expression of CD1d antigen on mature T cells. J Leukoc Biol. 2001;69(2):207-14.

39. Kang SS, Kim HJ, Jang MS, Moon S, In Lee S, Jeon JH, et al. Gene expression profile of human peripheral blood mononuclear cells induced by Staphylococcus aureus lipoteichoic acid. Int Immunopharmacol. 2012;13(4):454-60.

40. Ragin MJ, Sahu N, August A. Differential regulation of cytokine production by CD1d-restricted NKT cells in response to superantigen staphylococcal enterotoxin B exposure. Infect Immun. 2006;74(1):282-8.

41. Wu H, Rodgers JR, Perrard XY, Perrard JL, Prince JE, Abe Y, et al. Deficiency of CD11b or CD11d results in reduced staphylococcal enterotoxin-induced T cell response and T cell phenotypic changes. J Immunol. 2004;173(1):297-306.

42. Goh B, Loo RW, McAloney RA, Goh MC. Diffraction-based assay for detecting multiple analytes. Anal Bioanal Chem. 2002;374(1):54-6.

43. Goh JB, Tam PL, Loo RW, Cynthia Goh M. A quantitative diffraction-based sandwich immunoassay. Anal Biochem. 2003;313(2):262-6.

44. Loo RW, Tam PL, Goh JB, Goh MC. An enzyme-amplified diffraction-based immunoassay. Anal Biochem. 2005;337(2):338-42.

45. Edwards DA. Transport effects on surface reaction arrays: biosensor applications. Math Biosci. 2011;230(1):12-22.

46. Wadhwa M, Thorpe R. Unwanted immunogenicity: lessons learned and future challenges. Bioanalysis. 2010;2(6):1073-84.

47. Gnanaprakasa TJ, Oyarzabal OA, Olsen EV, Pedrosa VA, Simonian AL. Tethered DNA scaffolds on optical sensor platforms for detection of hipO gene from Campylobacter jejuni. Sensors Actuators B Chem. 2011;156(1):304-11.

48. Lin Y, Fu Q, Zhu J, Miller JM, Van Eyk JE. Development of a qualitative sequential immunoassay for characterizing the intrinsic properties of circulating cardiac troponin I. Clin Chem. 2010;56(8):1307-19.

49. Borisenko V, Hu W, Tam P, Chen I, Houle JF, Ausserer W. Diffractive optics technology: a novel detection technology for immunoassays. Clin Chem. 2006;52(11):2168-72.

50. De Santis ML, Hammamieh R, Das R, Jett M. Adipocyte-fatty acid binding protein induces apoptosis in DU145 prostate cancer cells. J Exp Therapeut Oncol. 2004;4(2):91-100.

51. Fishelevich R, Malanina A, Luzina I, Atamas S, Smyth MJ, Porcelli SA, et al. Ceramide-dependent regulation of human epidermal keratinocyte CD1d expression during terminal differentiation. J Immunol. 2006;176(4):2590-9.

52. Sikder H, Zhao Y, Balato A, Chapoval A, Fishelevich R, Gade P, et al. A central role for transcription factor C/EBP-beta in regulating CD1d gene expression in human keratinocytes. J Immunol. 2009;183(3):1657-66.

53. Zhao Y, Fishelevich R, Petrali JP, Zheng L, Anatolievna MA, Deng A, et al. Activation of keratinocyte protein kinase $C$ zeta in psoriasis plaques. J Investig Dermatol. 2008;128(9):2190-7.

54. Hammamieh R, Chakraborty N, Das R, Jett M. Molecular impacts of antisense complementary to the liver fatty acid binding protein (FABP) 
mRNA in DU 145 prostate cancer cells in vitro. J Exp Therapeut Oncol. 2004;4(3):195-202.

55. Behar SM, Dascher CC, Grusby MJ, Wang CR, Brenner MB. Susceptibility of mice deficient in CD1D or TAP1 to infection with Mycobacterium tuberculosis. The Journal of experimental medicine. 1999;189(12):1973-80.

56. Sousa AO, Mazzaccaro RJ, Russell RG, Lee FK, Turner OC, Hong S, et al. Relative contributions of distinct MHC class I-dependent cell populations in protection to tuberculosis infection in mice. Proc Natl Acad Sci U S A. 2000;97(8):4204-8.

57. Koch M, Stronge VS, Shepherd D, Gadola SD, Mathew B, Ritter G, et al. The crystal structure of human CD1d with and without alpha-galactosylceramide. Nat Immunol. 2005;6(8):819-26.

58. Kang SJ, Cresswell P. Calnexin, calreticulin, and ERp57 cooperate in disulfide bond formation in human CD1d heavy chain. The Journal of biological chemistry. 2002;277(47):44838-44.

59. Kumar H, Belperron A, Barthold SW, Bockenstedt LK. Cutting edge: CD1d deficiency impairs murine host defense against the spirochete, Borrelia burgdorferi. J Immunol. 2000;165(9):4797-801.

60. Kronenberg M. Toward an understanding of NKT cell biology: progress and paradoxes. Annu Rev Immunol. 2005;23:877-900.

\section{Submit your next manuscript to BioMed Central and take full advantage of:}

- Convenient online submission

- Thorough peer review

- No space constraints or color figure charges

- Immediate publication on acceptance

- Inclusion in PubMed, CAS, Scopus and Google Scholar

- Research which is freely available for redistribution 